\title{
Frequency-Dependent Parameterized Macromodeling of Integrated Inductors
}

\author{
F. Passos, E. Roca, R.Castro-López, F.V. Fernández \\ Instituto de Microelectrónica de Sevilla \\ IMSE, CNM (CSIC, Universidad de Sevilla) \\ Seville, Spain \\ \{moreira,eli,castro,francisco.fernandez\}@imse-cnm.csic.es
}

\author{
Y. Ye, D. Spina, T. Dhaene \\ Department of Information Technology \\ IBCN, Ghent University \\ Ghent, Belgium \\ \{yinghao.ye,dspina,tdhaene\}@intec.ugent.be
}

\begin{abstract}
Integrated inductors are one of the most important passive elements in radio frequency design, due to their wide usage in wireless circuits, such as transceivers. Typically, electromagnetic simulators are used in order to estimate the inductors performance with high accuracy as a function of the inductor geometrical and electrical parameters. Such simulations offer high-accuracy, but are computationally expensive and extremely time consuming. In this paper a frequency-dependent parameterized macromodeling technique is adopted in order to overcome this problem. The proposed approach offers a high degree of automation, since it is based on sequential sampling algorithms, high efficiency and flexibility: a continuous frequency-domain model is given for each value of the chosen inductors parameters in the design space.
\end{abstract}

Keywords-integrated inductors; surrogate modeling; parametric macromodels; $R F$ design

\section{INTRODUCTION}

One of the new and most popular trends in electronics industry nowadays is the Internet of Things (IoT): connecting and identifying any physical object to the internet. Therefore, new forms of communication between people and things are established, spreading the world of information and communication. For the IoT to become a reality, it is necessary to embed short-range mobile transceivers into several everyday items. Therefore, the design and development of wireless/radio frequency $(\mathrm{RF})$ devices is attracting a great deal of attention.

Among the different types of RF devices, the design of integrated inductors has drawn a strong interest in both the integrated circuit and electronic automation design communities, since they are essential components of modern $\mathrm{RF}$ circuits [1]. Integrated inductors are broadly used in filters, voltage controlled oscillators (VCOs), power amplifiers (PAs), low noise amplifiers (LNAs) and impedance matching networks. However, obtaining high-performance integrated inductors is one of the most challenging tasks in RF design. Typically, designers rely on electromagnetic (EM) simulators, which are the most accurate performance evaluators for integrated inductors. EM simulations are really accurate, but computationally intensive and, therefore, time consuming. With nowadays demand for short time-to-market, adopting iterative EM simulations during the design stage of integrated inductors is becoming unaffordable. The scope of the proposed

This work was supported in part by the TEC2013-45638-C3-3-R Project (funded by the Spanish Ministry of Economy and Competitiveness and ERDF), in part by the P12-TIC-1481 Project (funded by Junta de Andalucía), and in part by the CSIC Project PIE 201350E058. contribution is to overcome the high computational time of EM simulations, while estimating the inductors performance parameters with accuracy and small computational effort.

Parametric macromodeling techniques are a reliable alternative to EM simulators, being able to model the complex behavior of EM systems based on the frequency or time and additional geometrical or electrical design parameters. Parametric macromodeling techniques have attracted much attention in the last few years [2], [3]. In this contribution, a frequency-dependent parameterized macromodel using a sequential sampling technique is adopted, which allows the evaluation of the inductor transfer function in a matter of milliseconds.

The proposed paper is organized as follows. Section II, presents the main characteristics of integrated inductors; Section III carefully describes the parameterized macromodeling technique adopted. In Section IV, the proposed model is validated and its accuracy is compared with respect to EM simulations. Finally, conclusions are drawn in Section V.

\section{INTEGRATED INDUCTORS}

Integrated inductors are devices typically defined by the geometrical parameters shown in Fig. 1: the outer diameter $D_{\text {out }}$, the inner diameter $D_{\text {in }}$, the spacing $S$ between turns, the turn width $W$ and the number of turns $N$. The most relevant inductor performance parameters are the equivalent inductance $\left(L_{e q}\right)$ and quality factor $(Q)$ for a given frequency value, which are defined as:

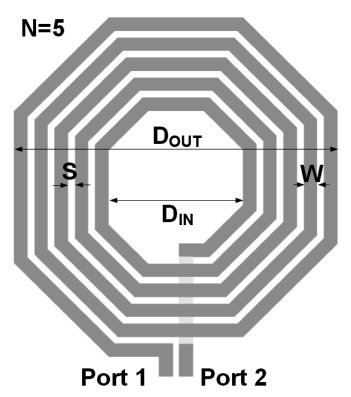

Fig. 1. Geometrical parameteres for an octagonal asymmetric spiral inductor. 


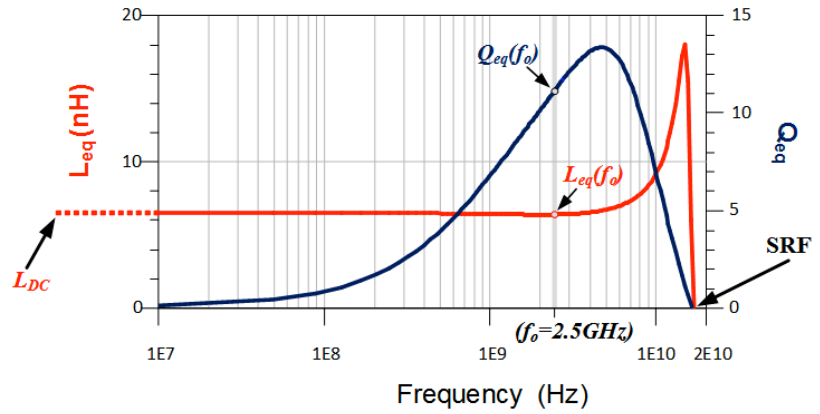

Fig. 2. Illustrating inductors performance parameters: inductance and quality factor vs frequency curves.

$L_{e q}(f)=\frac{\operatorname{Im}\left[Z_{e q}(f)\right]}{2 \pi f}$

$Q(f)=\frac{\operatorname{Im}\left[Z_{e q}(f)\right]}{\operatorname{Re}\left[Z_{e q}(f)\right]}$

where, $f$ is the frequency and $Z_{e q}$ is the equivalent input impedance, which can be obtained by the scattering parameters (S-parameter) of the two-port representation of the inductor. A graphical description of $L_{e q}, Q$ and self-resonance frequency $(S R F)$ is given in Fig. 2.

\section{PARAMETRIC MACROMODELING TECHNIQUE}

The main purpose of this work is to evaluate the inductor Sparameters by means of a parameterized macromodel $\boldsymbol{R}(s, \boldsymbol{g})$, where $s$ is the Laplace variable and $\boldsymbol{g}$ is a vector of geometrical or electrical parameters. By modeling the S-parameters, a designer can later obtain the impedance matrix, admittance matrix, inductance, or quality factor in a straightforward way.

Therefore, a specific parameterized macromodeling technique is adopted [3], which computes a stable and passive model of the inductor S-parameters with respect to the chosen variables $\boldsymbol{g}$ by interpolating root macromodels in a state-space form. This modeling technique is based on a combination of rational identification, positive interpolation schemes, and sequential sampling techniques [3] - [4]. For each particular combination of the design parameters $\boldsymbol{g}=\hat{\boldsymbol{g}}$ in the entire design space, the parameterized macromodel gives an equivalent statespace model as:

$$
R(s, \hat{g})=C(\hat{g})(s I-A(\hat{g}))^{-1} B(\hat{g})+D(\hat{g})
$$

which represents the inductor S-parameters over the frequency range of interest. Note that $\boldsymbol{I}$ is the identity matrix. Hence, the model describes the relationship between the inductor design parameters and the corresponding S-parameters over a continuous range of frequencies in a state-space form,

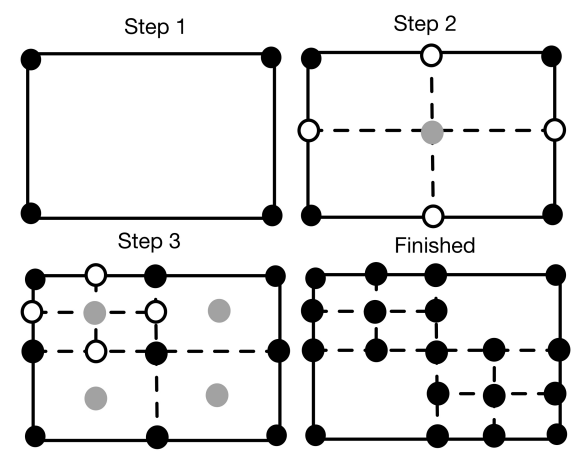

Fig. 3. Demonstrating the automated sequential sampling technique.

preserving the inductor fundamental physical properties, such as stability and passivity [3] - [5]. In the following, the procedure required to build the desired parameterized macromodel is described in detail.

\section{A. Model building phase}

The starting point of the adopted macromodeling technique [3] is to evaluate the inductor S-parameters by means of EM simulators over a set of samples for the frequency and the chosen design parameters: $\boldsymbol{S}\left(s_{j}, \boldsymbol{g}_{k}\right)$ for $j=1, \ldots, J$ and $k=1, \ldots, K$. Starting from these initial data samples, a corresponding set of frequency-dependent rational models is built by means of the Vector Fitting (VF) technique [4], [5]. A pole-flipping scheme is used to enforce stability [4], while passivity assessment and enforcement can be accomplished using standard techniques [5]. After this initial phase, a set of stable and passive frequency dependent rational models, called root macromodels, is computed. Now, the set of root macromodels must be parameterized with respect to the design variables $\boldsymbol{g}$ by means of stability and passivity preserving interpolations schemes.

Note that the technique [3] is a local scalable macromodeling method. Indeed, starting from the initial data samples, the design space is divided into cells, and a local parameterized macromodel is associated to every cell, which is a sub-domain of the entire design space. Afterwards, amplitude and frequency scaling system coefficients are computed for the root macromodels of each cell by means of an optimization procedure and then properly parameterized by positive interpolation operators [6]. Adopting the scaling interpolation method proposed in [3] considerably enhances the modeling capability of standard interpolation schemes and allows to accurately model highly dynamic systems with less number of EM simulations. Hence, a parameterized macromodel is obtained by a combination of root macromodels and corresponding amplitude and frequency scaling coefficients, using positive interpolation schemes in order to preserve stability and passivity over the complete design space [3].

The main computational cost to build the desired model is the initial requirement of EM simulations. In order to efficiently choose the initial samples and the corresponding cells, the automated sampling technique [2] is adopted. Once an appropriate error measure is chosen, the technique [2] sequentially selects samples in the design space which allow building an accurate model (see Fig. 3). Hence, the building 
phase of the parameterized macromodel is fully automated. The proposed modeling strategy is summarized in Fig. 4.

\section{B. Parameterized macromodeling of integrated inductors}

In this work, suitable parameterized macromodels are built for inductors with different number of turns $N$ (e.g. one model for inductors with two turns, another for inductors with three turns, etc.). The design parameters are $\mathrm{D}_{\text {in }}$ and $\mathrm{W}$, see Fig. 1, which vary in the following ranges: $\mathrm{D}_{\mathrm{in}} \in[10,300] \mu \mathrm{m}, \mathrm{W} \in[5$, $25] \mu \mathrm{m}$, under the constraint that $D_{\text {out }}<400 \mu \mathrm{m}$. The latter constraint ensures that the area of the integrated inductor is not too large, see Fig. 1. The spacing between the different turns of the inductor is maintained fixed at $2.5 \mu \mathrm{m}$. The inductor Sparameters are evaluated over 17 frequency samples in the range $[0.0001,2.7] \mathrm{GHz}$. The number of frequency samples used has a direct impact on the accuracy/efficiency trade-off of the entire modeling process: using more frequency points give more information on the behavior of the integrated inductor, but it will lead to an increased computational time, since EM simulations must be performed for each frequency sample.

The maximum absolute error adopted for the proposed modeling technique and the automated sampling scheme was set to $-50 \mathrm{~dB}$ for the magnitude of the S-parameters. Table I show the number of samples used to build the parameterized macromodels for inductors with different number of turns. Table I shows the number of root macromodels computed for each inductor (see "Samples to Build Model"), and the number of samples used to verify the accuracy of the parameterized macromodel over the design space chosen by the automated sampling strategy (see "Samples to Test Model"). It is possible to observe that for lower number of turns, namely one or two, the model creation does not require many EM simulations; this number increases with respect to the number of turns due to the complexity of the inductor behavior. However, for six and seven turns, the number of required EM simulations decreases mainly due to the chosen constraint $D_{\text {out }}<400 \mu \mathrm{m}$ : the feasible design space for higher number of turns is reduced.

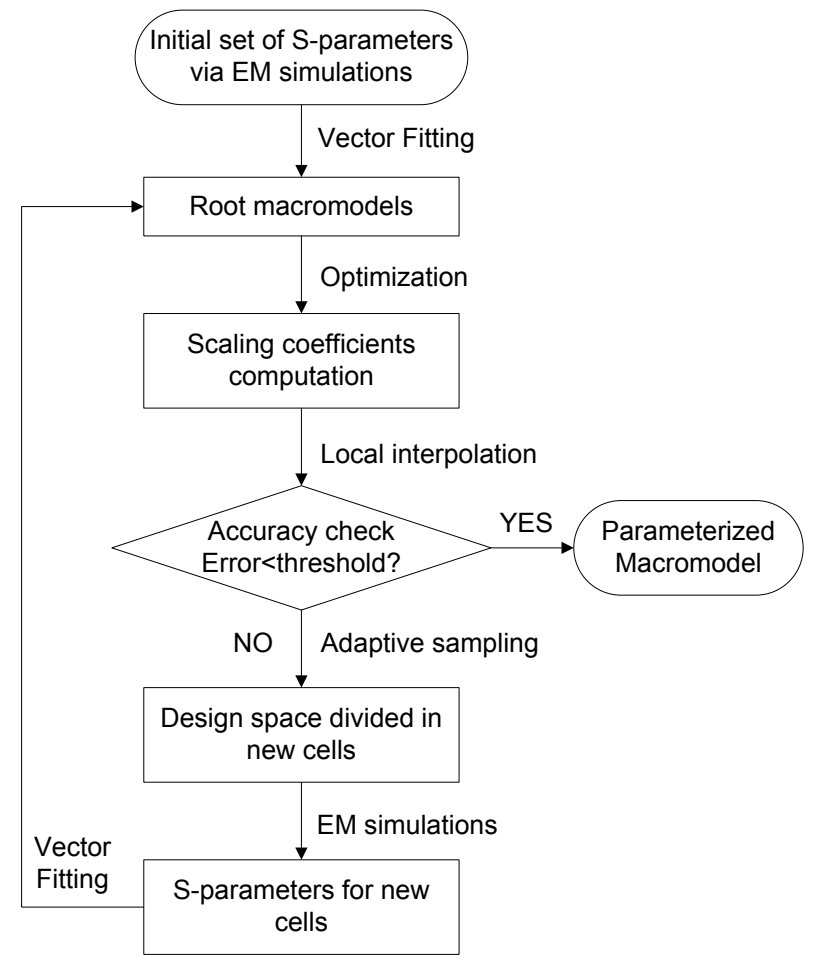

Fig. 4. Flowchart for the creation of the proposed parameterized macromodel.
TABLE I. SAMPLES NEEDED TO BUILD THE MODELS.

\begin{tabular}{|c|c|c|c|c|c|c|c|}
\hline & \multicolumn{7}{|c|}{ Inductor turns } \\
\cline { 2 - 8 } & $\mathbf{1}$ & $\mathbf{2}$ & $\mathbf{3}$ & $\mathbf{4}$ & $\mathbf{5}$ & $\mathbf{6}$ & $\mathbf{7}$ \\
\hline $\begin{array}{c}\text { Samples to } \\
\text { Build Model }\end{array}$ & 71 & 75 & 116 & 126 & 127 & 113 & 98 \\
\hline $\begin{array}{c}\text { Samples to } \\
\text { Test Model }\end{array}$ & 56 & 55 & 87 & 98 & 95 & 85 & 76 \\
\hline
\end{tabular}

\section{MODEL VALIDATION}

In this Section the accuracy of the parameterized macromodels computed for inductors with different number of turns is studied.

Fig. 5 shows the S-parameters for an inductor with $\mathrm{N}=6$, $\mathrm{D}_{\text {in }}=97.05 \mu \mathrm{m}$ and $\mathrm{W}=10.05 \mu \mathrm{m}$ computed with the corresponding parameterized macromodel over the 17 frequency samples used to build the model and via EM simulations: it can be observed that the accuracy of the model is quite good. However, one of the advantages of such modeling approach is the ability of estimating the performance parameters of an integrated inductor for frequency samples which have not been used to build the model, since the parameterized macromodel is continuous over the chosen frequency range. Fig. 6 shows a comparison of the Sparameters for an inductor with $N=2, \mathrm{D}_{\text {in }}=181.4 \mu \mathrm{m}$ and $\mathrm{W}=15.75 \mu \mathrm{m}$ and the corresponding results by means of EM simulations over a set of frequency samples which have not been used to build the macromodel (validation frequencies) and over the 17 frequency samples used to build the macromodel (modeling frequencies). Again, the model proves to be very accurate even for frequencies which have not been used for the model calculation.

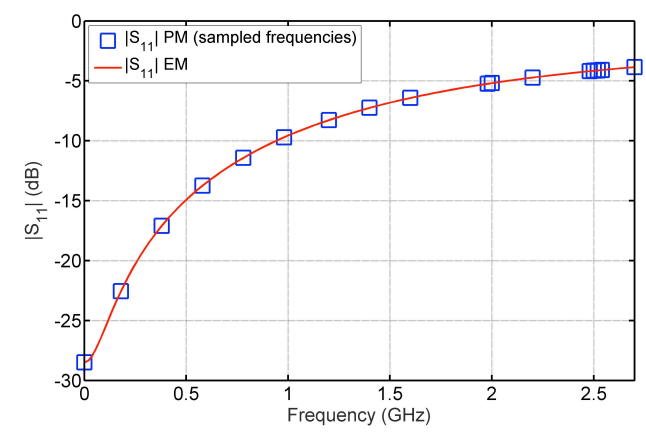

Fig. 5. Magnitude of the element $S_{11}$ of a six turn inductor given by the parametric macromodel (blue squares) compared with respect to EM simulations (red line).

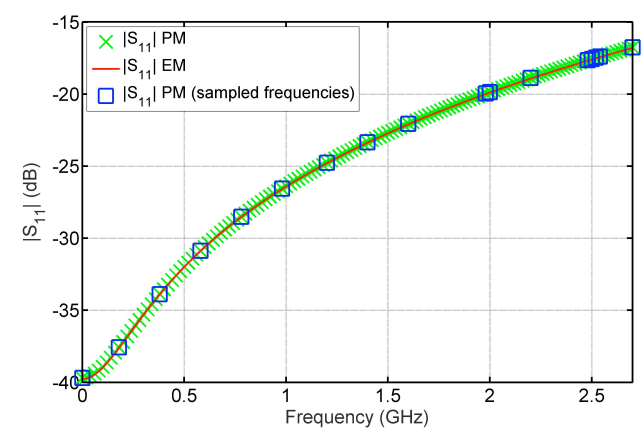

Fig. 6. Magnitude of the element $S_{11}$ of a two turn inductor given by the parametric macromodel over the modeling frequencies (blue squares) and validation frequencies (green exes) compared with respect to EM simulations (red line). 
By modeling the S-Parameters it is also possible to directly calculate $\mathrm{L}$ and $\mathrm{Q}$ for the entire range of frequencies: Fig. 7 shows the inductance and quality factor of an inductor with $\mathrm{N}=2, \mathrm{D}_{\mathrm{in}}=261.1$ and $\mathrm{W}=9.25$ obtained with the computed model and by means of EM simulation.

Finally, in order to verify the model accuracy over the entire design space, an extensive study over a wide number of integrated inductors is performed at $2.5 \mathrm{GHz}$, which is a standard frequency of the industrial, scientific and medical (ISM) radio band. Hence, a grid of 1089 inductors was generated for each number of turns (7623 inductors in total), covering the entire design space. However, the proposed analysis has been limited to the 6184 inductors out of the total 7623 which satisfied the chosen area constraint $D_{\text {out }}<400 \mu \mathrm{m}$. The validation was performed for all inductors at $2.5 \mathrm{GHz}$ and is shown in Fig. 8. It is possible to observe that the maximum error for the elements of the S-parameter matrix is less than $50 \mathrm{~dB}$ for each tested inductor. The extensive study performed sustains the fact that the computed models are indeed very accurate and the technique successfully allowed a proper modeling of integrated inductors.

\section{CONCLUSIONS}

In this work a parameterized macromodeling technique has been applied for the modeling of the S-parameter of integrated inductors. The accuracy of the computed models has been verified against the results of EM simulations. An extensive study has been performed by testing 6184 inductors over a wide range of geometries proving therefore the validity of the models on the entire design space. Starting from the Sparameters, the calculation of the inductors performance parameters is trivial and the models computed are continuous over the chosen frequency range.
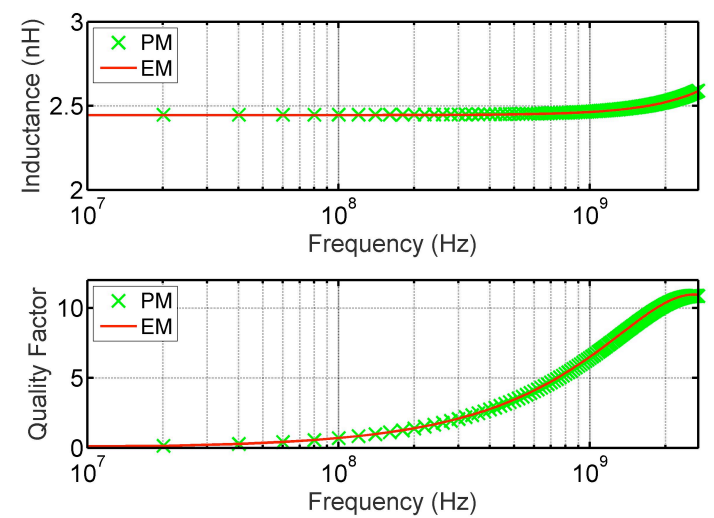

Fig. 7. Inductance and quality factor of a two turn inductor given by the parametric macromodel (green exes) over the validation frequencies compared with respect to EM simulations (red line).

\section{REFERENCES}

[1] A. Niknejad, Electromagnetics for High-Speed Analog and Digital Communication Circuits: Cambridge University Press, 2007.

[2] K. Chemmangat, F. Ferranti, T. Dhaene, L. Knockaert, "Scalable models of microwave system responses using sequential sampling on unstructured grids", International journal of numerical modeling: Electronic networks, devices and fields, vol. 27, pp. 122-137, 2014.

[3] F. Ferranti, L. Knockaert, T. Dhaene, and G. Antonini, "Parametric macromodeling based on amplitude and frequency scaled systems with guaranteed passivity", International Journal of Numerical Modelling: Electronic Networks, Devices and Fields, vol. 25, issue 2, pp. 139-151, March/April 2012.

[4] B. Gustavsen and A. Semlyen, "Rational approximation of frequency domain responses by vector fitting," IEEE Transactions on Power Delivery, vol. 14, no. 3, pp. 1052-1061, 1999.

[5] T. Dhaene, D. Deschrijver, and N. Stevens, "Efficient Algorithm for Passivity Enforcement of S-Parameter Based Macromodels," IEEE Transactions on Microwave Theory and Techniques, vol. 57, no. 2, pp. 415-420, 2009.

[6] G. Allasia, "Simultaneous interpolation and approximation by a class of multivariate positive operators", Numerical Algorithms, vol. 34, issue 2, pp. 147-158, 2003.
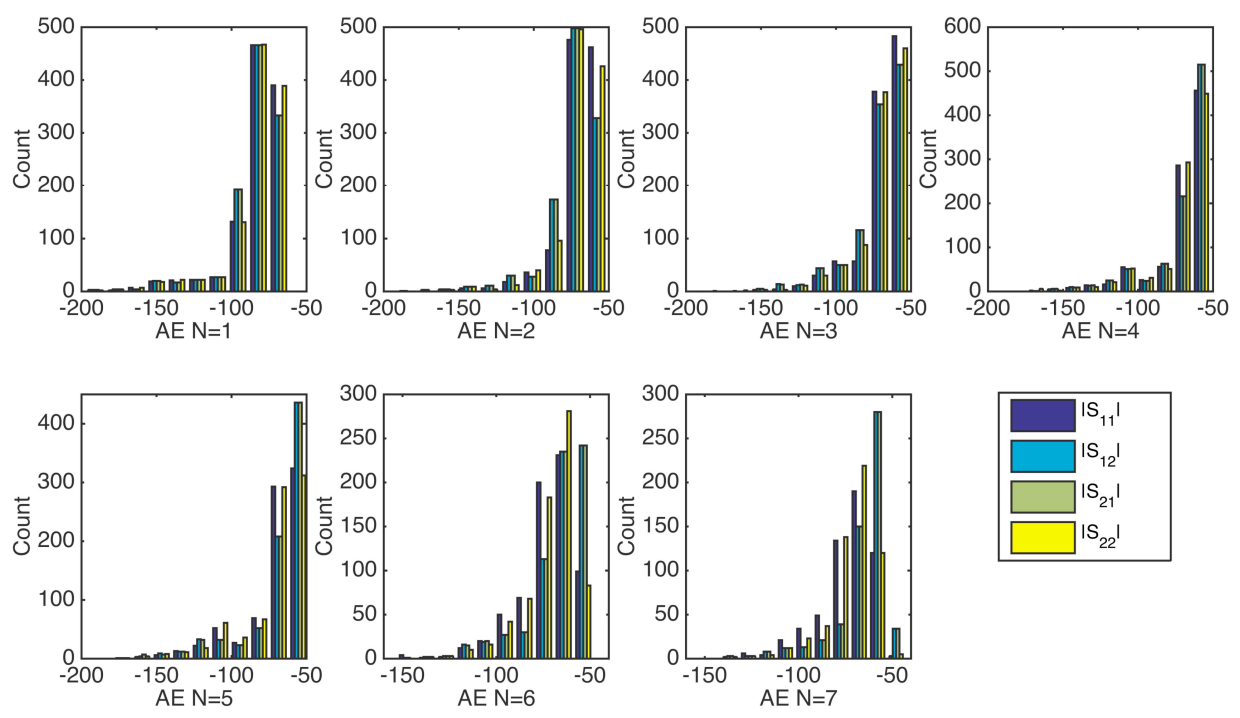

Fig. 8. Extensive study of the model accuracy using 6184 inductors. Absolute error (in $\mathrm{dB}$ ) vs. number of inductors (count) for each number of turns (N). 